\title{
Development of a robust transient expression screening system in protoplasts of Cannabis
}

\author{
L. Matchett-Oates ${ }^{1,2}$ (1) Ehab Mohamaden ${ }^{1}$ - G. C. Spangenberg ${ }^{1,2} \cdot$ N. O. I. Cogan ${ }^{1,2}$ \\ Received: 8 November 2020 / Accepted: 23 March 2021 / Published online: 17 May 2021 / Editor: Ted Klein \\ (C) The Author(s) 2021
}

\begin{abstract}
Transient expression systems in mesophyll protoplasts have been utilised in many plant species as an indispensable tool for gene function analysis and efficacious genome editing constructs. However, such a system has not been developed in Cannabis due to the recalcitrant nature of the plant to tissue culture as well as its illegal status for many years. In this study, young expanding leaves from aseptic in vitro Cannabis explants were used for protoplast isolation. Factorial designs were used to optimise variables in viable protoplast isolation and transient expression of GFP, with a range analyses performed to determine, and quantify, significantly impacting variables. Viable protoplast yields as high as $5.7 \times 10^{6}$ were achieved with $2.5 \%(w / v)$ Cellulase R-10, $0.3 \%(w / v)$ Macerozyme R-10 and $0.7 \mathrm{M}$ mannitol, incubated for $16 \mathrm{~h}$. As indicated by the transient expression of GFP, efficiency reached $23.2 \%$ with $30 \mu \mathrm{g}$ plasmid, $50 \% \mathrm{PEG}, 1 \times 10^{6}$ protoplasts and a transfection duration of $20 \mathrm{~min}$. Application of the optimised protocol for protoplast isolation was successfully evaluated on three subsequent unrelated genotypes to highlight the robustness and broad applicability of the developed technique.
\end{abstract}

Keywords Cannabis $\cdot$ Protoplasts $\cdot$ Transient $\cdot$ Transformation

\section{Introduction}

Cannabis sativa L. (Cannabis) is a highly polymorphic, wind pollinated herb originating in China where evidence of its cultivation dates back to $4000 \mathrm{BC}$ (Zuardi 2006). Recent interest in the medicinal properties of phytocannabinoids produced by Cannabis has led to increased legalisation around the world, along with a growing medicinal industry (ProCon.org 2021). Recently, chromosomal resolution of the Cannabis genome was published along with other genetic resources for identification of important cannabinoid biosynthesis genes (Grassa et al. 2018; Laverty et al. 2019). Next generation sequencing has also started to unravel the complexity of the Cannabis genome and transcriptome atlas (Braich et al. 2019). Progress in the genetic tools for manipulation and

L. Matchett-Oates

lennon.matchett-oates@agriculture.vic.gov.au

1 Agriculture Victoria, the Centre for AgriBioscience, AgriBio, 5 Ring Road, Bundoora, Victoria 3083, Australia

2 School of Applied Systems Biology, La Trobe University, Bundoora, Victoria 3086, Australia analysis of genes have prompted research into Cannabis, but currently, Cannabis remains a recalcitrant species to deliver biotechnology tools to. Transgenic hairy root cultures of Cannabis have been performed previously using agroinfiltration of vectors with the GUS reporter gene (Wahby et al. 2013). However, hairy root cultures only offer the accumulation of metabolites within the root structures (Gurunani et al. 2015) making this transformational technique unsuitable for biotechnological applications seeking phytocannabinoids, which accumulate in the female floral tissues. Transformation of hemp callus cultures with a foreign Escherichia coli gene, manA, has previously been successful using Agrobacterium tumefaciens with a success rate of $31 \%$ (Feeney and Punja 2003). PCR analysis of callus cultures confirmed stable gene integration with up to four T-DNA copies being integrated into the genome; however, plantlet regeneration was unsuccessful.

With the recent release of the chromosomal assembly and high-density linkage map of the Cannabis genome (Grassa et al. 2018; Laverty et al. 2019), identification of genes involved in cannabinoid biosynthesis is now relatively straightforward, with benchmark standards being developed for genetic engineering of these genes (Matchett-Oates et al. 2020.). However, to date, no procedures for the evaluation of genes 
through functional screening exist, due to the recalcitrant nature of Cannabis in vitro and the difficulty in obtaining transgenic explants (Feeney and Punja 2017). In addition, the illegal status of cannabis during the time in which biotechnology has emerged and has caused Cannabis to fall behind in genetic improvement studies. Genetic transformation is used for the study of gene function and genetic improvement in plants. Protocols for many non-model species, in which regeneration of transgenic plants has not been achieved, have been developed for screening genome editing constructs. To date, only brief protocol outlines for protoplast isolation have been reported (Jones 1979; Morimoto et al. 2007), with no protoplast transient expression protocols existing for Cannabis. Creating stable transformants is expensive and time consuming making this approach for large scale evaluation of cannabinoid biosynthesis genes limiting. Utilising protoplasts ability to transiently express DNA constructs has allowed for highthroughput transient screening of genes in Arabidopsis (Marion et al. 2008), gene-silencing in barley (Douchkov et al. 2005) and functional analysis of newly isolated genes in tobacco (Fischer and Hain 1995). Such studies are yet to be conducted on Cannabis making the need for an efficient transient expression system in protoplasts of high importance.

Protoplasts are osmotically fragile due to the lack of a cell wall from enzymatic digestion, allowing for the transfer of DNA constructs through the plasma membrane using common methods such as PEG-mediated transfection (Yoo et al. 2007), Agrobacterium infiltration (Clough and Bent 1998) and biolistic bombardment (Vain et al. 1993). Transient gene expression from plant protoplasts has widely been used to study cell death related processes (Chen et al. 2015), developmental studies (Sheen 2001), subcellular localisation of proteins ( $\mathrm{Su}$ et al. 2010) and expression of foreign genes (Zhang et al. 2011). During isolation, optimum conditions such as osmotic balance, enzyme concentration and digestion time need to be established by optimising all variables contributing to yield and viability. The use of reporter genes in transient assays is often used due to the non-toxic nature, stability and ease of detection allowing for protein localisation and interactions studies (Leffel et al. 1997) making protoplasts an important tool in gene analysis.

The evaluation of transiently expressed shared terpene biosynthesis genes between Cannabis, and other species has been reported (Reed and Osbourn 2018; Smirnoff 2019). However, Cannabis contains a unique set of enzymes in the cannabinoid biosynthesis pathway, which synthesise phytocannabinoids that are only produced in this species. The elucidation of the cannabinoid biosynthesis genes in the draft Cannabis genome provided the first complete look into the multiple pathways involved (Van Bakel et al. 2011). Identification and characterisation of the first unique enzyme in the cannabinoid pathway, OLS, have evolved from identification of an unknown polyketide synthase (Raharjo et al. 2004). Initially characterised as a novel polyketide synthase-olivetol synthase (Taura et al. 2009), later, the mechanism for olivetol production requiring olivetol acid cyclase, a DABB protein (Gagne et al. 2012), was identified and correctly characterised. Similarly, identification and functional studies on the oxidocyclases, tetrahydrocannabinolic acid synthase (THCAS) and cannabidiolic acid synthase (CBDAS) have evolved as technological advancements into molecular cloning techniques, and DNA sequencing has improved. Tetrahydrocannabinol (THC), one of the major constituents in Cannabis, was originally presumed to be formed by the isomerisation of cannabidiolic acid (CBDA) (Shoyama et al. 1975). Protein sequencing and PCR cloning of tetrahydrocannabinolic acid (THCA) confirmed that THC was produced from cannabigerolic acid (CBGA) by THCAS (Sirikantaramas et al. 2004). Identification of CBDAS (Taura et al. 1996) occurred shortly after with a similar strategy used for gene identification (Taura et al. 2007).

Genetic engineering of Cannabis offers the opportunity to produce higher levels of cannabinoids with tailor-made chemical profiles for medicinal applications. However, the recalcitrant nature of Cannabis and the absence of methods to efficiently generate transgenic plants currently makes transient expression systems important to understand the molecular regulatory mechanisms responsible for cannabinoid biosynthesis, which remain largely unknown. Prior to this report, a transient expression system in Cannabis protoplasts did not exist, making the characterisation of unique gene function speculative. In this present study, an efficient protoplast isolation and transient expression system using Cannabis leaf mesophyll protoplasts are reported. Optimisation to obtain a high yield of viable protoplasts and PEG-mediated transfection of the protoplasts using fluorescent reporter genes is also reported. Furthermore, due to the outbreeding highly heterozygous nature of Cannabis, strain-dependent response variation is expected; this protoplast isolation and transient expression systems is shown to be suitable to many strains, highlighting the applicability for future protoplast and transient expression studies.

\section{Materials and Methods}

Plant Material and Expression Vectors All research was performed under Medicinal Cannabis Research Licence (RL011/ 18) and Permit (RL01118P4) issued through the Department of Health (DoH), Office of Drug Control (ODC) Australia. A specific genotype (C. sativa) with high THC content $(25 \%)$ was used for optimisation within this study. Aseptic plantlets were derived from apical meristems of mature vegetative mother plants, which were sterilised by a 1-min $80 \%$ ethanol wash, washed three times with sterile water, disinfected with $10 \%(v / v)$ sodium hypochlorite (White King, Melbourne, 
Australia) solution containing $4.5 \%$ active chlorine for $15 \mathrm{~min}$ and finally washed three times with sterile water. Apical meristems were cultured in root induction media containing 1/2 Murashige and Skoog salts and vitamins (MS; Murashige and Skoog 1962) (Duchefa Biochemie, Haarlem, The Netherlands), $1 \%$ sucrose $(w / v)$ (Sigma-Aldrich, St. Louis, MO), $1 \%$ agar ( $w / v)$ (Duchefa Biochemie) and $1 \mathrm{mg}$ $\mathrm{L}^{-1}$ Indole-3-butyric acid (IBA) (Sigma-Aldrich) adjusted to $\mathrm{pH} 5.7$ prior to autoclaving at $121^{\circ} \mathrm{C}$ for $15 \mathrm{~min}$. The apical meristems were cultured at $26^{\circ} \mathrm{C}$ under lighting of $74 \mu \mathrm{mol}$ $\mathrm{m}^{-2} \mathrm{~s}^{-1}$ supplied by fluorescent lamps for $18 \mathrm{~h}$ per day. The green fluorescent protein (GFP) expression vector pDONR221-GFP was constructed from pDONR221 (Invitrogen, Carlsbad, CA) through BP Clonase reaction by the insertion of the attB $1-\mathrm{CaMV} 35 \mathrm{D}-$ p_turboGFP(D)_AtuNos-t-attB2 cassette (Supplementary Information).

\section{Protoplast Isolation, Purification and Quantification} Protoplasts were isolated from well rooted, 1- to 2 mo-old plantlets with young leaves cut into 0.5 - to 1.0 -mm-thin strips (Fig. $1 a-d$ ) and incubated in a Petri dish containing digestion media comprising of 1,2 and $2.5 \%(w / v)$ Cellulase Onozuka R-10 (Yakult Honsha Co., Ltd., Tokyo, Japan); 0.3, 0.4 and $0.5 \%(w / v)$ Macerozyme R-10 (Yakult Honsha Co.); 0.3, 0.5 and 0.7 M mannitol (Sigma-Aldrich, St Louis, MO); $20 \mathrm{mM}$ MES (Sigma-Aldrich, St Louis, MO); 20 mM KCl (SigmaAldrich, St Louis, MO); and $10 \mathrm{mM} \mathrm{CaCl}_{2}$ (Sigma-Aldrich), $\mathrm{pH}$ adjusted to 5.8 and filter-sterilised using a $0.22-\mu \mathrm{m}$ filter (Sigma-Aldrich). Leaf strips were incubated in the dark at $28^{\circ} \mathrm{C}$ without agitation for between 8 and $24 \mathrm{~h}$ with each digestion replicated three times. Following digestion, the cellular suspension was mechanically filtrated through a $70-\mu \mathrm{m}$ mesh (Corning, NY) into a sterile $50-\mathrm{mL}$ polypropylene centrifuge tube (Corning, NY) and centrifuged at $700 \times g$ for 10 min. Following centrifugation, the resulting supernatant was discarded, and the pellet resuspended with $3 \mathrm{~mL}$ of W5 $(5 \mathrm{mM}$ glucose, $5 \mathrm{mM} \mathrm{KCl}, 10 \mathrm{mM}$ MES, $125 \mathrm{mM} \mathrm{CaCl}_{2}, 154 \mathrm{mM}$ $\mathrm{NaCl}, \mathrm{pH} 5.8$ ), transferred to a $15-\mathrm{mL}$ round bottomed tube and $3 \mathrm{~mL} 20 \%(w / v)$ sucrose added and centrifuged again. Protoplasts were collected from the interphase (Fig. 1e) into a fresh $15-\mathrm{mL}$ round bottom tube and $3 \mathrm{~mL}$ W5 added and centrifuged again with the supernatant removed. Finally, the pellet was resuspended in $1 \mathrm{~mL} \mathrm{W5}$, and $100 \mu \mathrm{L}$ of the resuspended protoplasts were diluted with $0.5 \mathrm{M}$ Evans Blue, and approximately 50 to 200 protoplasts were counted using a haemocytometer under a light microscope. The viability of the protoplasts was calculated by (viable protoplasts/total number of protoplasts) $\times 100 \%$.

PEG-Mediated Transfection Isolated protoplasts were divided into aliquots of $5 \times 10^{5}$ or $1 \times 10^{6}$ and centrifuged at $700 \times g$ for $10 \mathrm{~min}$ with the supernatant removed. PEG4000 solutions
$(20 \%, 30 \%, 40 \%$ and $50 \%(w / v))$ (Sigma-Aldrich) were prepared by dissolving in $\mathrm{ddH}_{2} \mathrm{O}$ containing $0.1 \mathrm{M} \mathrm{Ca}\left(\mathrm{NO}_{3}\right)_{2}$ $4 \mathrm{H}_{2} \mathrm{O}$ and $0.4 \mathrm{M}$ Mannitol (Sigma-Aldrich). A volume of $100 \mu \mathrm{L}$ transformation buffer $\left(15 \mathrm{mM} \mathrm{MgCl}_{2}, 0.5 \mathrm{M}\right.$ Mannitol, $0.1 \%(w / v)$ MES, $\mathrm{pH} 5.7)$ was added to the protoplasts and gently mixed. Plasmid concentrations (5, 10 and 30 $\mu \mathrm{g}$ ) in $60 \mu \mathrm{L}$ of $\mathrm{ddH}_{2} \mathrm{O}$ were added to the protoplast solution and gently mixed followed by addition of $150 \mu \mathrm{L}$ of warmed $\left(42^{\circ} \mathrm{C}\right)$ PEG4000 solutions. The protoplast solution was mixed gently and incubated in the dark at room temperature $\left(22 \pm 1^{\circ} \mathrm{C}\right.$ ) for between 10 and $60 \mathrm{~min}$. The transfection reaction was stopped by the addition of $5 \mathrm{~mL}$ W5 dropwise, followed by a further $5 \mathrm{~mL}$ in a gentle stream. The protoplast mixture was centrifuged once more at $700 \times g$ for $10 \mathrm{~min}$, removing the supernatant and the addition of $150 \mu \mathrm{L}$ W5 followed by incubation in the dark at room temperature for $48 \mathrm{~h}$. The expression of GFP was observed under a fluorescence microscope (OLYMPUS CKX53, Tokyo, Japan) (excitation emission wavelengths 470 to $490 \mathrm{~nm}, 510 \mathrm{~nm}$ ).

FACS Analysis The protoplasts were analysed using Influx ${ }^{\mathrm{TM}}$ FACS instrument (BD Biosciences, Franklin Lakes, NJ) fitted with a $200-\mu \mathrm{m}$ nozzle using W5 buffer as a sheath fluid. The sheath pressure was set at 4 psi, and the sample pressure was set at 5 psi. A 466-nm Coherent Sapphire Solid state laser was used for excitation, and emission was measured using a 517/ $18 \mathrm{~nm}$ band-pass filter for GFP. The photomultiplier tube voltage was set at $16.41 \mathrm{~V}$ for forward scatter, $20.59 \mathrm{~V}$ for side scatter and $40.37 \mathrm{~V}$ for GFP. The threshold value for event detection was set at 0.3 on side scattering. For analysis, a gate was set using PEG-transfected control protoplasts. Frequency of GFP expressing cells was recorded, and data was processed using BD FACSTM Software v1.0.0.650 (BD Biosciences).

Statistical Analysis Experimental data was statistically analysed using Minitab 19 Statistical Software (Version 19, State College, PA) and R Studio (Version 1.1.453, RStudio, Inc., Boston, MA).

\section{Results}

Mesophyll Protoplast Isolation From Cannabis Leaf Tissue Initially, protoplasts were generated from rapidly expanding leaves from 1- to 2-mo-old in vitro plantlets that were used as the source material (Fig. 1 $a, b$ ). Briefly, plantlets cultured at $26^{\circ} \mathrm{C}$ in $1 / 2 \mathrm{MS}$ medium with $5 \mu \mathrm{M}$ IBA were used for mesophyll protoplast isolation. One gram of young leaf material was cut into 0.5 - to $1-\mathrm{mm}$ strips and immediately transferred into digestion medium in the dark without agitation at $28^{\circ} \mathrm{C}$ for 8 to $24 \mathrm{~h}$ (Fig. $1 a-d)$. Protoplasts were collected through a 
Figure 1. (a) In vitro 1 - to 2-moold rooted explants grown on Murashige and Skoog medium containing $1 \mathrm{mg} \mathrm{L}^{-1}$ indole-3butyric acid suitable for protoplast isolation. Scale bar $=2 \mathrm{~cm}$. (b) Healthy Cannabis sativa L. leaves suitable for protoplast isolation. Scale bar $=2 \mathrm{~cm}$. (c) Cannabis sativa L. leaves sliced into 0.5 - to 1 -mm-thin strips with a fresh razor blade and placed in media. Scale bar $=2 \mathrm{~cm}$. (d) Cannabis sativa L. $16 \mathrm{~h}$ post digestion in the dark at $28^{\circ} \mathrm{C}$ without shaking. Scale bar $=2$ cm. (e) Purified Cannabis sativa L. protoplast interphase after density gradient centrifugation.
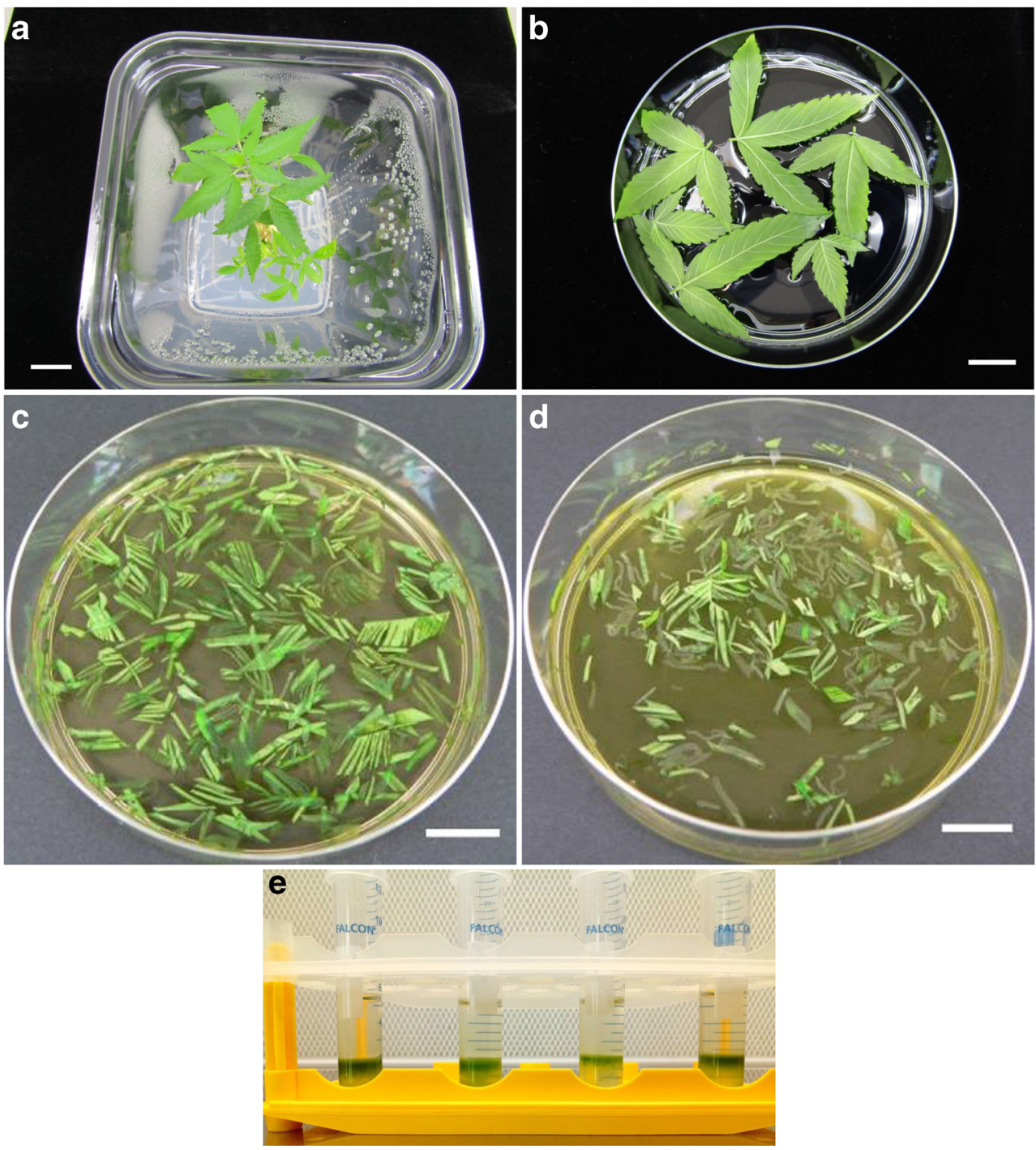

$70-\mu \mathrm{m}$ nylon mesh, centrifuged and washed as previously described.

\section{Single Factor Effect on Mesophyll Protoplast Isolation To op-} timise mesophyll protoplast isolation, enzyme and mannitol concentrations and enzymatic digestion time were adjusted. Mesophyll protoplasts were isolated from young, expanding leaves with variable digestion parameters, outlined in Table 1. Total protoplast yields ranged from $1.2 \times 10^{6}$ to $9.2 \times 10^{6}$ per gram of fresh leaf weight with the highest yield obtained from treatment 7 . Protoplast viability ranged from 39 to $79 \%$, with the highest viability obtained from treatment 9. An L9 $\left(3^{4}\right)$ orthogonal test (Table 1) was designed to identify statistically significant variables to optimise protoplast isolation, with a range analysis performed to predict the optimal combination of the variables. The range analysis of the means between the factors and levels (Table 2), calculated Delta scores (mean score of the highest range minus the lowest mean range) for enzymolysis time ( $\mathrm{R}$ 2.21) and Cellulase R-10 (R 1.56) significantly influencing protoplast isolation, with Macerozyme R-10 (R 1.44) and mannitol (R 1.23) proving less significant. Analysis of each level calculated response (k1-3) from the range analysis (Table 2), the Duncans multiple range test (Duncans) assigned the best possible combination of each factor as A3:B1:C3:D2. The calculated theoretical best combination was already performed in treatment 7 , with an average yield of $5.7 \times 10^{6}$ viable protoplasts.

Increasing Cellulase-R10 concentration significantly increased protoplast yield within multilevel factors (Fig. 3), from $1.31(1 \%)$ to $2.86(2.5 \%) \times 10^{6}$ viable protoplasts. This trend, of increasing concentrations greatly increasing yields, was not observed for the other variables, where applicable. Macerozyme R-10 effected protoplast yields significantly at $0.3 \%$ producing $2.93 \times 10^{6}$ viable protoplasts, whereas at $0.4 \%$ and $0.5 \%$, this decreased to $1.50 \times 10^{6}$ and $2.14 \times 10^{6}$ viable protoplasts, respectively. A concentration of $0.7 \mathrm{M}$ mannitol was more substantial in producing $2.89 \times 10^{6}$ viable protoplasts, whereas $0.3 \mathrm{M}$ produced $2.01 \times 10^{6}$, which 
Table 1. Results from the orthogonal L9 $\left(3^{4}\right)$ array for Cannabis sativa $\mathrm{L}$. protoplast isolation optimization

\begin{tabular}{|c|c|c|c|c|c|c|c|}
\hline \multirow[t]{2}{*}{ Treatment combination } & \multicolumn{4}{|l|}{ Factors } & \multirow{2}{*}{$\begin{array}{l}\text { Total yield } \\
\times 10^{6}(\mathrm{~g} \mathrm{FW})\end{array}$} & \multirow{2}{*}{$\begin{array}{l}\text { Yield of viable } \\
\times 10^{6}(\mathrm{~g} \mathrm{FW})\end{array}$} & \multirow{2}{*}{$\begin{array}{l}\text { Viability } \\
(\%)\end{array}$} \\
\hline & A & $\mathrm{B}$ & $\mathrm{C}$ & $\mathrm{D}$ & & & \\
\hline $\mathrm{T} 1$ & $1(1.0 \%)$ & $1(0.3 \%)$ & $1(0.3 \mathrm{M})$ & $1(8 \mathrm{~h})$ & $1.8 \pm 0.5$ & $1.3 \pm 0.3$ & $71 \pm 3.5$ \\
\hline $\mathrm{T} 2$ & $1(1.0 \%)$ & $2(0.4 \%)$ & $2(0.5 \mathrm{M})$ & $2(16 \mathrm{~h})$ & $2.5 \pm 0.5$ & $1.5 \pm 0.2$ & $63 \pm 10.4$ \\
\hline $\mathrm{T} 3$ & $1(1.0 \%)$ & $3(0.5 \%)$ & $3(0.7 \mathrm{M})$ & $3(24 \mathrm{~h})$ & $2.0 \pm 0.1$ & $1.2 \pm 0.1$ & $59 \pm 3.8$ \\
\hline $\mathrm{T} 4$ & $2(2.0 \%)$ & $1(0.3 \%)$ & $2(0.5 \mathrm{M})$ & $3(24 \mathrm{~h})$ & $3.8 \pm 0.3$ & $1.8 \pm 0.1$ & $47 \pm 1.5$ \\
\hline $\mathrm{T} 5$ & $2(2.0 \%)$ & $2(0.4 \%)$ & $3(0.7 \mathrm{M})$ & $1(8 \mathrm{~h})$ & $2.6 \pm 0.1$ & $1.8 \pm 0.2$ & $68 \pm 6.4$ \\
\hline T6 & $2(2.0 \%)$ & $3(0.5 \%)$ & $1(0.3 \mathrm{M})$ & $2(16 \mathrm{~h})$ & $5.3 \pm 0.3$ & $3.6 \pm 0.2$ & $68 \pm 4.0$ \\
\hline $\mathrm{T} 7$ & $3(2.5 \%)$ & $1(0.3 \%)$ & $3(0.7 \mathrm{M})$ & $2(16 \mathrm{~h})$ & $7.8 \pm 1.0$ & $5.7 \pm 1.0$ & $72 \pm 5.9$ \\
\hline $\mathrm{T} 8$ & $3(2.5 \%)$ & $2(0.4 \%)$ & $1(0.3 \mathrm{M})$ & $3(24 \mathrm{~h})$ & $3.1 \pm 0.2$ & $1.2 \pm 0.01$ & $39 \pm 1.8$ \\
\hline T9 & $3(2.50 \%)$ & $3(0.5 \%)$ & $2(0.5 \mathrm{M})$ & $1(8 \mathrm{~h})$ & $2.2 \pm 0.2$ & $1.7 \pm 0.1$ & $79 \pm 2.7$ \\
\hline
\end{tabular}

(A) Cellulase R-10 concentration $(w / v),(B)$ Macerozyme R-10 concentration $(w / v),(C)$ Mannitol concentration, $(D)$ enzymolysis time, T1-9 different treatment combinations, $1,2,3$ three factor levels

performed greater than $0.5 \mathrm{M}$, which produced $1.67 \times 10^{6}$ viable protoplasts (Table 3). Enzymolysis time of $16 \mathrm{~h}$ produced a two-fold increase in viable protoplasts, with $3.61 \times$ $10^{6}$ compared to a shorter digestion time of $8 \mathrm{~h}\left(1.58 \times 10^{6}\right)$ or a longer digestion time of $24 \mathrm{~h}\left(1.39 \times 10^{6}\right)$.

\section{Transient Transfection of Cannabis Mesophyll Protoplasts To} establish the first transient expression system, pDONR221GFP was used to study the effects of plasmid, PEG and protoplast concentration, including incubation time on transfection efficiency. The transfection efficiency reached $23.2 \%$ in treatment $12(30 \mu \mathrm{g}$ plasmid, 50\% PEG $(w / v), 20 \mathrm{~min}$ of incubation and $1 \times 10^{6}$ protoplasts), with the lowest recorded transfection rate of $5.78 \%$ in treatment $5(10 \mu \mathrm{g}$ plasmid, $20 \%$ PEG $(w / v), 20 \mathrm{~min}$ incubation time and $1 \times 10^{6}$ protoplasts).

\section{Single Factor Effect on Mesophyll Protoplast Transfection} Factors with multiple levels across variables (Table 3) were designed to optimise plasmid concentration, PEG

Table 2. Range analysis of L9 Cannabis sativa L. protoplast isolation orthogonal array

\begin{tabular}{lllll}
\hline & $\mathrm{A}$ & $\mathrm{B}$ & $\mathrm{C}$ & $\mathrm{D}$ \\
\hline $\mathrm{k} 1$ & 1.31 & 2.93 & 2.01 & 1.58 \\
$\mathrm{k} 2$ & 2.40 & 1.50 & 1.67 & 3.61 \\
$\mathrm{k} 3$ & 2.86 & 2.14 & 2.89 & 1.39 \\
Range & 1.56 & 1.44 & 1.23 & 2.22 \\
Optimum combination & $\mathrm{A} 3$ & $\mathrm{~B} 1$ & $\mathrm{C} 3$ & $\mathrm{D} 2$ \\
\hline
\end{tabular}

$\mathrm{k} 1, \mathrm{k} 2$, and $\mathrm{k} 3$ indicate mean viable protoplast $\times 10^{6}(\mathrm{~g} \mathrm{FW})$ at the 1,2 , and 3 levels. The larger the range value, the greater the influence of the factors on test results. All values expressed as $\times 10^{6}$

(A) cellulase R-10 concentration ( $w / v),(B)$ Macerozyme R-10 concentration $(w / v),(C)$ Mannitol concentration, $(D)$ enzymolysis time concentration $(w / v)$, transfection time and protoplast concentration variables in transfection efficiency. Mesophyll protoplasts were transfected using the PEG-mediated transfection protocol described previously (Fig. 2) with transfection efficiency ranging from 5.78 to $23.20 \%$ with the highest average transfection rate, $23.20 \%$, obtained from treatment 12 . Within multilevel factors, increasing plasmid concentration greatly increased protoplast transfection within efficiencies between 10 and $30 \mu \mathrm{g}$ (11.11 to $17.29 \%)$. Increasing PEG concentration from 20 to $50 \%$ saw increases in transfection efficiency, with $50 \%$ achieving $16.23 \%$ on average, compared to $20 \%$ PEG $(w / v)$, which saw $10.93 \%$ transfection efficiency. Incubation time of 30 min gave the highest average transient efficiency of $15.82 \%$. Similarly, a 20 -min incubation resulted in $16.61 \%$ transfection efficiency. Incubation of 10 and 60 min saw a reduction of transfection efficiency, 12.02\% and $13.25 \%$, respectively. Protoplast density of $5 \times 10^{5}$ achieved higher transient efficiencies on average compared to $1 \times 10^{6}$, with $14.63 \%$ and $11.72 \%$ efficiency, respectively. From the range analysis of the means (Table 4), the calculated Delta values of plasmid concentration (R 6.18\%) and PEG concentration ( $\mathrm{R} 5.3 \%$ ) greatly influence transfection efficiency, with transfection time (R 3.8\%) and protoplasts density (R $2.91 \%$ ) proving less significant.

\section{Discussion}

Protoplasts offer a versatile experimental system, with transient expression systems widely applied in Arabidopsis (Yoo et al. 2007), rice (Wang et al. 1988) and tobacco (Töpfer et al. 1988) to analyse gene expression and function and to deliver genetic improvements in plants. Crucial for the development of an efficient transient expression system for genome editing constructs is the routine generation of high-quality protoplasts 
Table 3. Results from the factorial array for Cannabis sativa L. protoplast transfection efficiency optimization

\begin{tabular}{llllll}
\hline Treatment combination & \multicolumn{2}{l}{ Factors } & & \multirow{2}{*}{ Transfection efficiency } \\
\cline { 2 - 4 } & $\mathrm{A}$ & $\mathrm{B}$ & $\mathrm{C}$ & $\mathrm{D}$ & $(\%)$ \\
\hline $\mathrm{T} 1$ & $1(5 \mu \mathrm{g})$ & $1(20 \%)$ & $1(10 \mathrm{~m})$ & $1(0.5)$ & $9.78 \pm 2.59$ \\
$\mathrm{~T} 2$ & $1(5 \mu \mathrm{g})$ & $2(30 \%)$ & $2(20 \mathrm{~m})$ & $1(0.5)$ & $14.35 \pm 2.48$ \\
$\mathrm{~T} 3$ & $1(5 \mu \mathrm{g})$ & $3(40 \%)$ & $3(30 \mathrm{~m})$ & $2(1)$ & $10.90 \pm 0.35$ \\
$\mathrm{~T} 4$ & $1(5 \mu \mathrm{g})$ & $4(50 \%)$ & $4(60 \mathrm{~m})$ & $2(1)$ & $9.41 \pm 0.48$ \\
$\mathrm{~T} 5$ & $2(10 \mu \mathrm{g})$ & $1(20 \%)$ & $2(20 \mathrm{~m})$ & $2(1)$ & $5.78 \pm 1.22$ \\
$\mathrm{~T} 6$ & $2(10 \mu \mathrm{g})$ & $2(30 \%)$ & $1(10 \mathrm{~m})$ & $2(1)$ & $6.20 \pm 0.47$ \\
$\mathrm{~T} 7$ & $2(10 \mu \mathrm{g})$ & $3(40 \%)$ & $4(60 \mathrm{~m})$ & $1(0.5)$ & $16.44 \pm 2.09$ \\
$\mathrm{~T} 8$ & $2(10 \mu \mathrm{g})$ & $4(50 \%)$ & $3(30 \mathrm{~m})$ & $1(0.5)$ & $16.08 \pm 1.35$ \\
$\mathrm{~T} 9$ & $3(30 \mu \mathrm{g})$ & $1(20 \%)$ & $3(30 \mathrm{~m})$ & $1(0.5)$ & $17.24 \pm 1.99$ \\
$\mathrm{~T} 10$ & $3(30 \mu \mathrm{g})$ & $2(30 \%)$ & $4(60 \mathrm{~m})$ & $1(0.5)$ & $13.88 \pm 0.33$ \\
$\mathrm{~T} 11$ & $3(30 \mu \mathrm{g})$ & $3(40 \%)$ & $1(10 \mathrm{~m})$ & $2(1)$ & $14.83 \pm 0.66$ \\
$\mathrm{~T} 12$ & $3(30 \mu \mathrm{g})$ & $4(50 \%)$ & $2(20 \mathrm{~m})$ & $2(1)$ & $23.20 \pm 0.61$ \\
\hline
\end{tabular}

(A) plasmid concentration, $(B)$ PEG concentration $(w / v),(C)$ experiment time, $(D)$ protoplast density $\left(\times 10^{6}\right), T 1$ 12 different treatment combinations, 1, 2, 3, 4 four factor levels and robust transfection protocols. Only two reports of isolated Cannabis protoplasts exist; however, the reports briefly discuss the methods for protoplast isolation with no data on protoplast yield and viability, or the data collection was not described in detail. Morimoto et al. (2007) digested Cannabis leaves with $1 \%$ Cellulase R-10, $0.2 \%$ Macerozyme R-10, $0.1 \%$ pectolyase $\mathrm{Y}-23$ and $0.4 \mathrm{M}$ mannitol at $30^{\circ} \mathrm{C}$ for $4 \mathrm{~h}$ with gentle agitation; however, no yield or viability data was given. Jones (1979) explored a range of enzyme combinations to produce protoplast from young and old leaf tissue, as well as callus, with concentrations between $1 \times 10^{3}$ and $1 \times 10^{5}$ reported. Protoplast isolation from the closely related species Humulus lupulus (hops) has previously been reported from cell suspension cultures. Several cell wall digesting enzyme mixtures were trialled with varying Cellulysin and Driselase concentrations in $0.4 \mathrm{M}$ mannitol osmoticum, with the highest yield of protoplasts (9.3 to $9.9 \times 10^{6}$ per gFW) obtained from $2 \%$ Cellulysin and $1 \%$ Driselase with 99 to $100 \%$ viability
Figure 2. (a) Visualisation of Cannabis sativa L. protoplasts under brightfield microscopy. Scale bar $=200 \mu \mathrm{m}$. $($ b $)$

Visualisation of Cannabis sativa L. protoplasts under fluorescence microscopy with GFP filter set. Scale bar $=50 \mu \mathrm{m}$. (c) Isolated protoplasts under fluorescence microscopy with GFP filter set. Scale bar $=200 \mu \mathrm{m} .($ d $)$ Isolated Cannabis sativa L. protoplasts under fluorescence microscopy with GFP filter set. Scale bar = $100 \mu \mathrm{m}$.
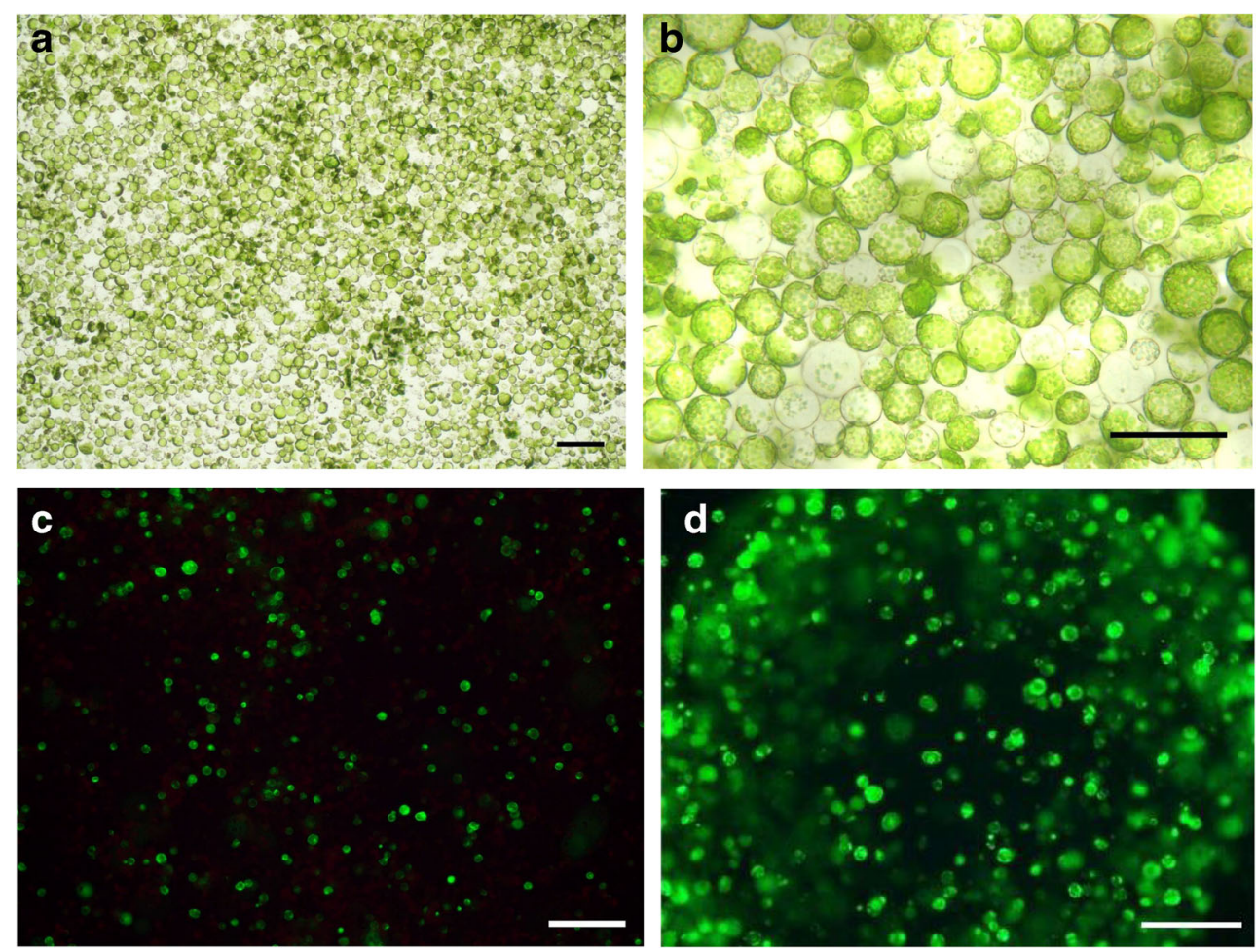
Table 4. Range analysis of factorial design for Cannabis sativa L. protoplast transfection efficiency

\begin{tabular}{lllll}
\hline & A & B & C & D \\
\hline k1 & 11.11 & 10.93 & 12.02 & 14.63 \\
$\mathrm{k} 2$ & 11.13 & 11.48 & 15.61 & 11.72 \\
$\mathrm{k} 3$ & 17.29 & 14.06 & 15.82 & \\
$\mathrm{k} 4$ & & 16.23 & 13.25 & \\
Range & 6.18 & 5.30 & 3.80 & 2.91 \\
\hline
\end{tabular}

$\mathrm{k} 1, \mathrm{k} 2, \mathrm{k} 3$, and $\mathrm{k} 4$ indicate mean transfection efficiency (\%) at the 1, 2, 3, and 4 levels. All values expressed as percentages

(A) plasmid concentration, $(B)$ PEG concentration $(w / v),(C)$ experiment time, $(D)$ protoplast density $\left(\times 10^{6}\right)$

reported (Furze et al. 1987). Heale et al. (Heale et al. 1989) reported leaf mesophyll isolation from the hop cultivar, Challenger, by firstly removing the epidermis followed by incubating in 4\% Cellulase, $0.3 \%$ Macerozyme and 2\% hemicellulose for $7 \mathrm{~h}$ on an orbital shaker protoplast yields between 4 and $7 \times 10^{5} \mathrm{~mL}^{-1}$ were achieved with viability ranging from 80 to $90 \%$ under optimal conditions. An efficient protocol for transient expression of LUC activity in rose (another member of the Order Rosales with Cannabis) leaves using Agrobacterium has also been reported (Lu et al. 2017). Recently, transient expression of $\beta$-glucuronidase (GUS) and GFP in a range of agroinfiltrated organs and tissues has been reported in Cannabis, with the protocol being optimised for hemp cultivars (Deguchi et al. 2020).

The development of an efficient protoplast transient expression system to screen genome editing constructs requires established protocols for the isolation of viable protoplasts and a competent transfection workflow, which until now have not been reported for Cannabis. With several factors affecting yield and viability of protoplasts and transfection efficiencies, this development of a routine system allows for significant advances to be made towards understanding and improving Cannabis cultivars. The method described here investigates the effect each variable has on protoplast isolation and transfection, with each being analysed for their significance.

To optimise protoplast isolation, Cellulase-R10, Macerozyme-R10, mannitol concentrations and digestion times were adjusted (Table 1). Under the optimum conditions, viable Cannabis leaf mesophyll protoplasts reached $5.7 \times 10^{6}$ protoplasts $\mathrm{g} \mathrm{FW}^{-1}$ (Table 1). The increase in Cellulase concentration to $2.5 \%(\mathrm{w} / \mathrm{v})$ showed significantly increased yields, $2.88 \times 10^{6}$, from the range analysis. Although $2.5 \%$ $(w / v)$ Cellulase concentration was the highest tested, increasing levels past a saturation point has been shown to decrease protoplast yields in tobacco (Kuriakose et al. 2012) and Magnolia (Shen et al. 2017). The optimal Macerozyme concentration was determined to be $0.3 \%(w / v)$, whilst an increase to $0.4 \%$ saw more than a $50 \%$ drop in protoplast recovery to
$1.50 \times 10^{6}$ calculated from the range analysis. For both enzymes, the increase in concentration at which viable protoplast yield decreases is presumably due to the influence those enzymes have on the membrane integrity. The application of the optimised protoplast isolation protocol to verify robustness on Cannbio-2, the alternative high THC strain and a high CBD strain produced a significant yield of viable protoplasts for subsequent transient expression experiments, demonstrating the robustness and versatility of the protocol described. The protocol produced similar protoplast viability, with Cannbio-2 averaging 79\%, equalling to the best performing treatment 9, and the high CBD strain producing higher levels of viability with $82 \%$ (Table 5). Although viable protoplast yield from these cultivars is proportionately fewer, the protocol has been optimised for a specific high yielding THC strain, with the calculated optimal conditions proven to be an advisory starting point for further optimisation on any chosen cultivar (Table 5).

Protoplasts lack of cell walls that require a stabilized environment, which is controlled by the osmotic gradient for proper osmolarity to sustain viable protoplasts (Sain et al. 2017). Mannitol is frequently used for it is inert metabolically and slowly diffuses through the cellular membrane (Chawla 2011). Mannitol concentration of $0.7 \mathrm{M}$ resulted in the highest yield, calculated by the range analysis, of $2.91 \times 10^{6}$, with lower concentrations of $0.3 \mathrm{M}$ and $0.5 \mathrm{M}$ yielding $2.02 \times$ $10^{6}$ and $1.67 \times 10^{6}$, respectively. Similar to enzyme concentration, increasing mannitol concentration, thus causing an imbalance in the osmoticum, decreases protoplast yield, as is seen with wheat (Jia et al. 2016) and pineapple (Priyadarshani et al. 2018). Enzymolysis time significantly affected viable protoplast yield, with digestions of 24-h yielding as low as $39 \%$ viability in treatment 8 , which most certainly caused by over digestion of the cell walls. Comparatively, 8 and $16 \mathrm{~h}$ were determined to yield significantly higher levels of viability, with $16 \mathrm{~h}$ shown to result in a larger concentration of viable protoplasts (Fig. 3) and the best digestion time for the release of viable protoplasts without over digestion.

The uptake of DNA through the plasma membrane of protoplasts for transient expression studies requires considerable concentrations of PEG acting in tandem with divalent cations (Maas and Werr 1989). With increasing concentrations of PEG, DNA hydration reduces causing structural changes

Table 5. Results of the mean Cannabis sativa L. protoplast isolation on multiple cultivars using optimised variable conditions

\begin{tabular}{lll}
\hline Cultivar & Yield of viable protoplasts $\left(\times 10^{6}\right)$ & Viability $(\%)$ \\
\hline Cannbio-2 & 1.97 & 79 \\
High THC & 1.82 & 72 \\
High CBD & 2.46 & 82 \\
\hline
\end{tabular}




\section{Effect of Cellulase Concentration On Viable Protoplast Yield}

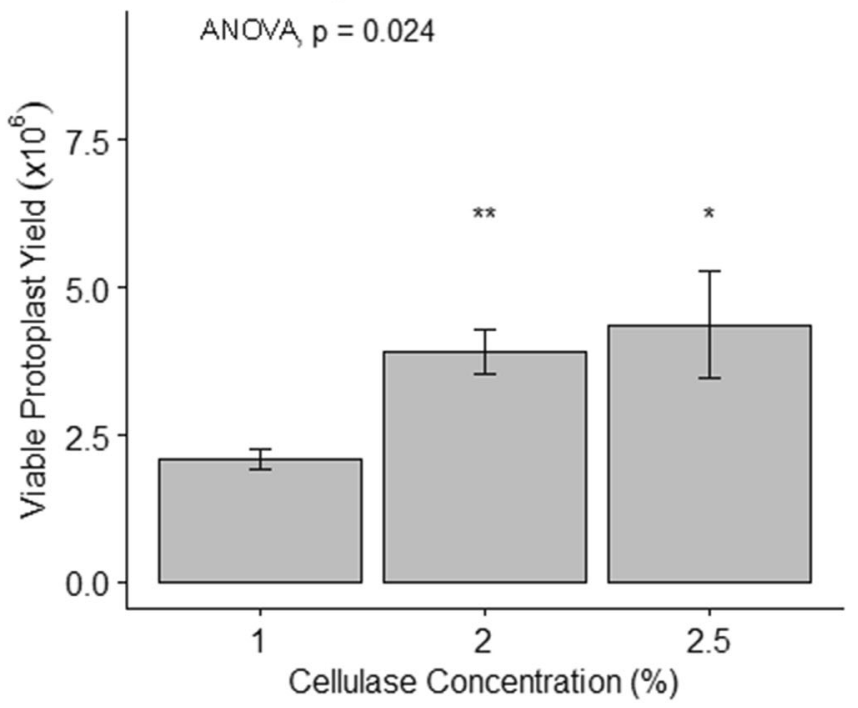

Effect of Mannitol Concentration On Viable Protoplast Yield

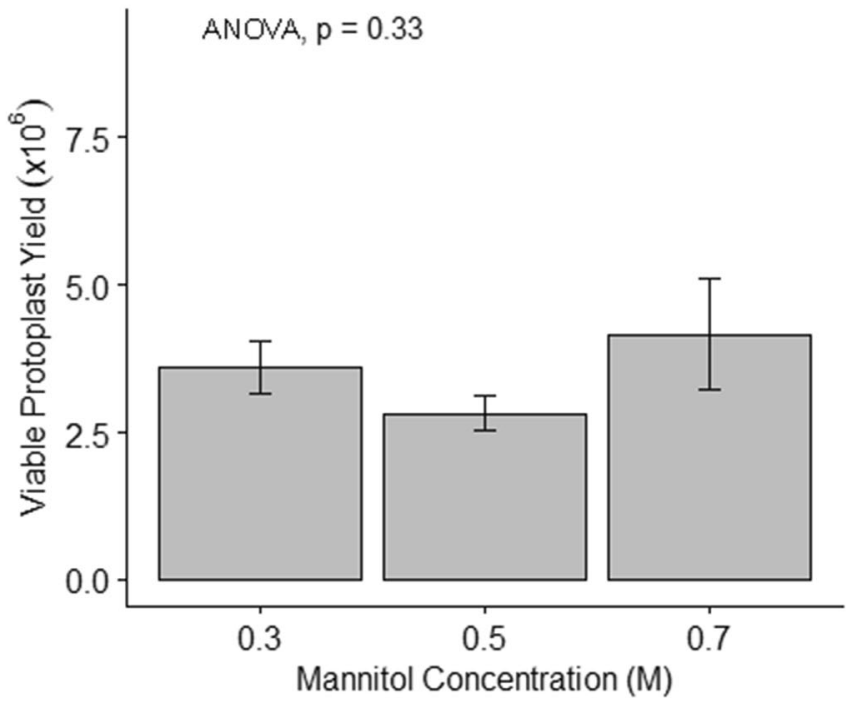

Effect of Macerozyme Concentration On Viable Protoplast Yield
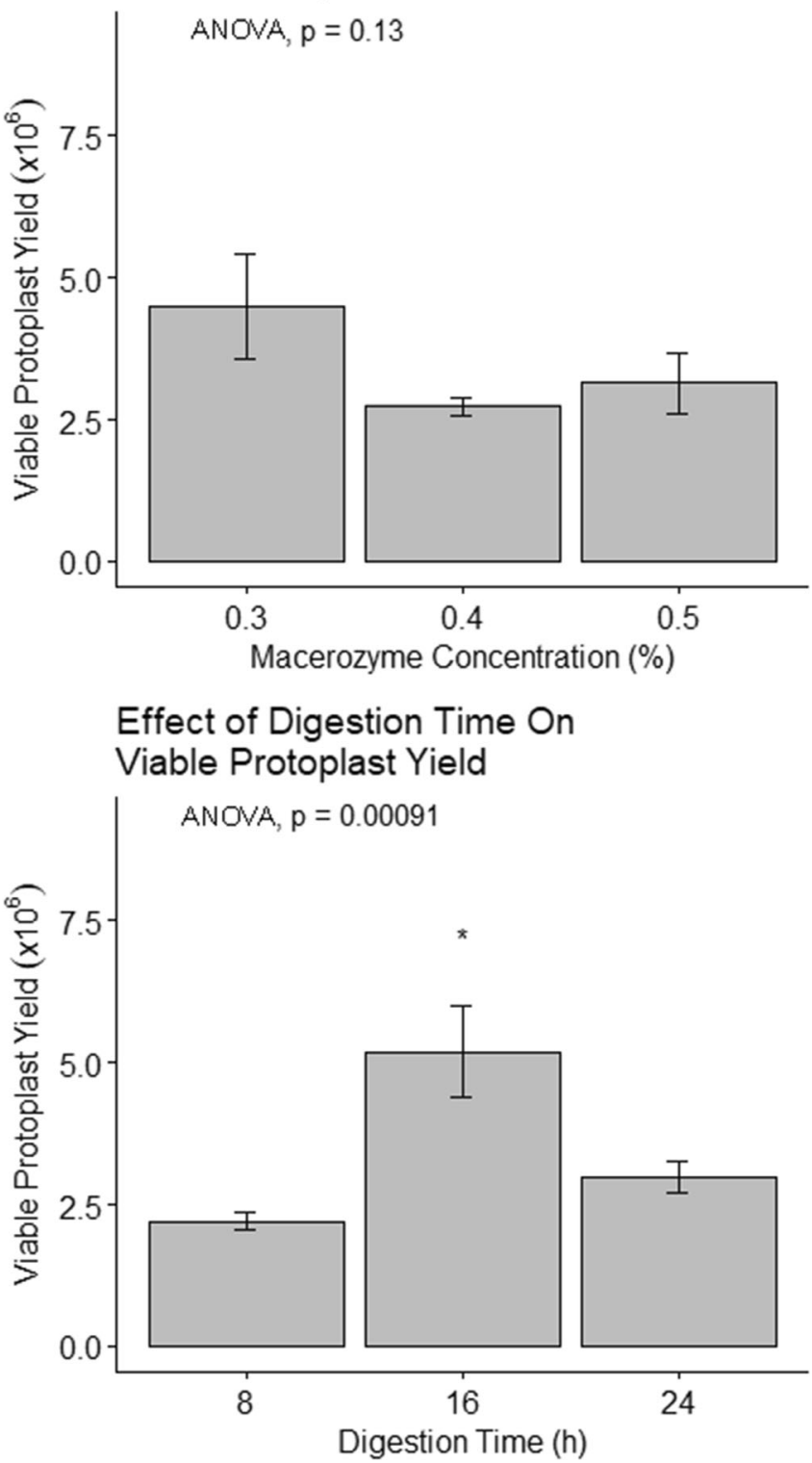

Figure 3. Effects of different factors on viable Cannabis sativa L. protoplast isolation yield. Bars represent standard errors (SE). Statistical significance was determined using a one-way ANOVA test $(* p<0.05, * * p<0.01)$.

and thus reducing the transfection efficiency (Saenger et al. 1986). In Cannabis protoplasts, transient expression efficiency at the highest investigated PEG concentration $(50 \%)$ achieved $16.23 \%$ (Fig. 4), which is a significantly higher concentration of PEG than has been previously reported in rice (Page et al. 2019) or pea (Nicolaisen and Poulsen 1993). Results show that increasing plasmid concentration from 5 to $10 \mu \mathrm{g}$ achieved similar transfection efficiencies, $11.11 \%$ and $11.13 \%$, respectively (Table 3 ). These results are inconsistent with findings in pepper (Jeon et al. 2007), pineapple (Priyadarshani et al. 2018) and Phaseolus (Nanjareddy et al. 2016) in which there is an approximate doubling in relevant transient expression efficiencies between these two concentrations. This suggests that Cannabis protoplasts require higher concentrations of plasmid to achieve increased levels of expression. Intermediate exposure time to high concentrations on PEG was shown to increase transfection efficiencies (Fig. 4), with the optimal exposure time determined to be $30 \mathrm{~min}$, achieving $15.82 \%$. Increasing the incubation time to 60 min saw a sharp decrease in transfection efficiency, falling to $12.02 \%$. The increased exposure to the high concentrations of PEG resulting in lower transfection efficiencies is expected due to DNA becoming less hydrated, with similar results found in carrot, rapeseed and soybean (Rasmussen and Rasmussen 1993). This protocol has been optimised for this particular cultivar (Fig. 4). This protocol provides a relevant 
Effect of Plasmid Concentration On

Viable Protoplast Yield

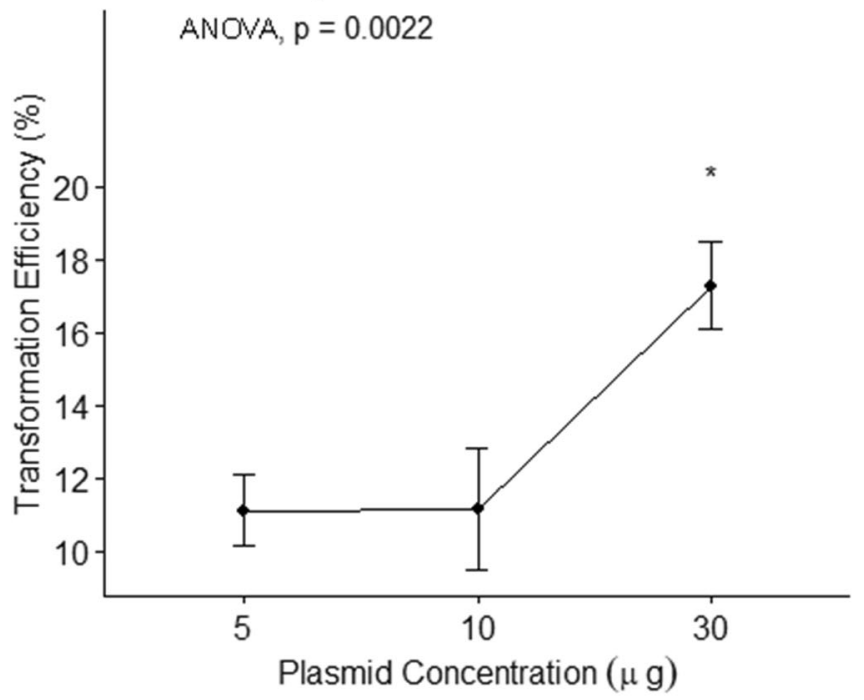

Effect of Protoplast Concentration On Viable Protoplast Yield

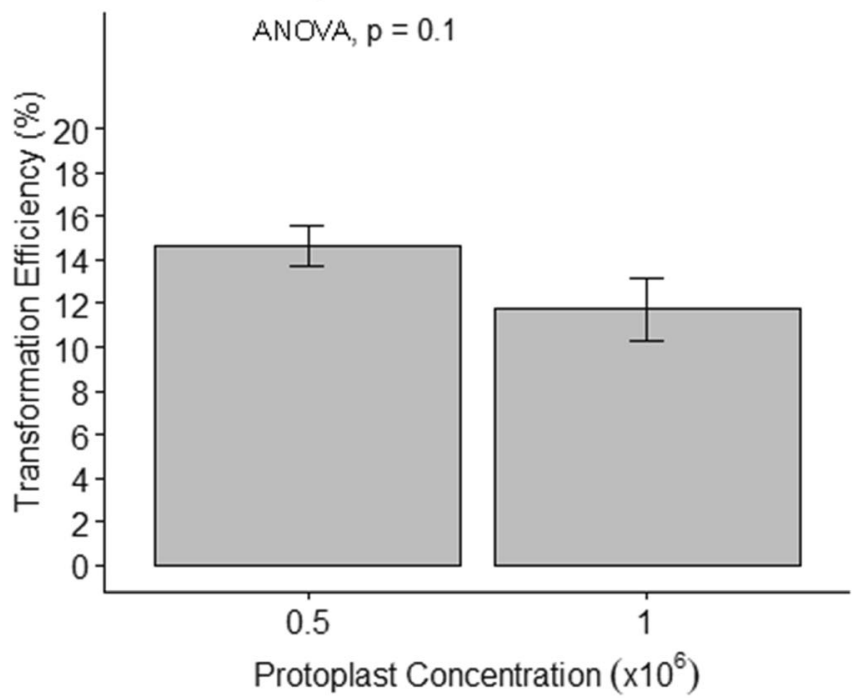

Effect of PEG Concentration On

Viable Protoplast Yield

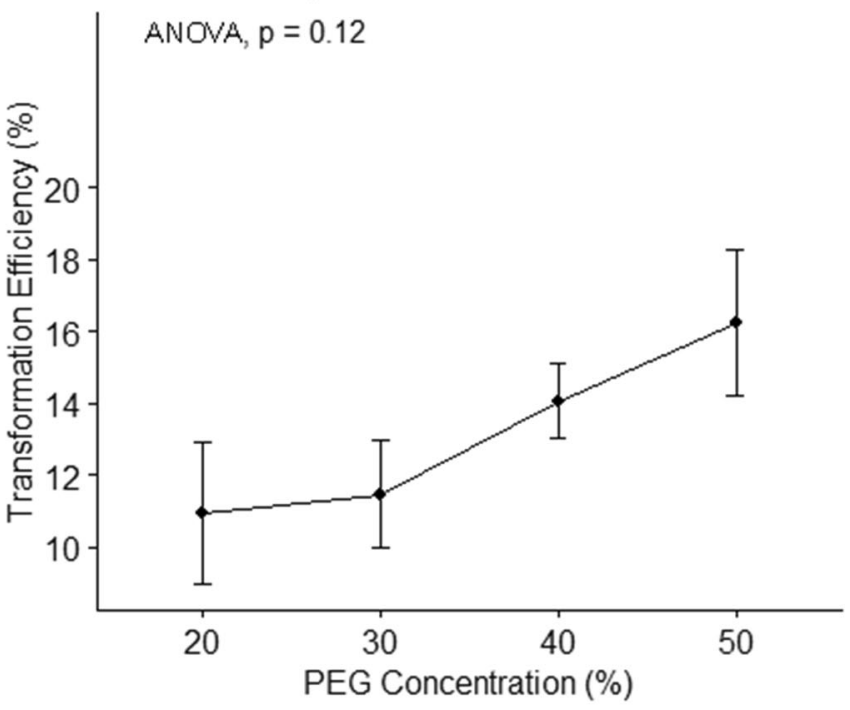

Effect of Transformational Time On Viable Protoplast Yield

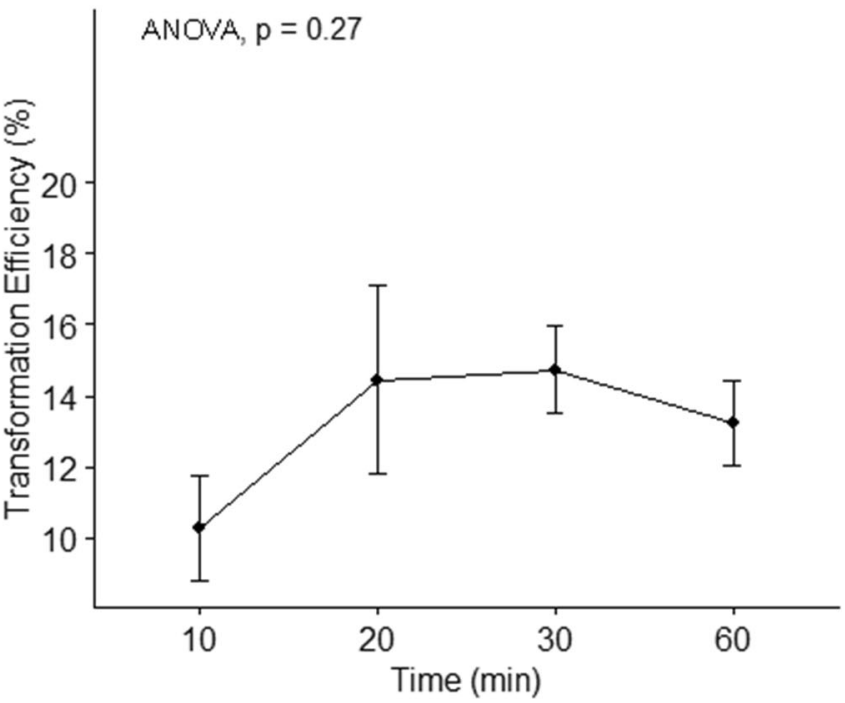

Figure 4. Effects of different factors on Cannabis sativa L. protoplast transfection efficiency. Bars represent standard errors (SE). Statistical significance was determined using a one-way ANOVA test $(* p<0.05)$.

starting point for optimisation regardless of genetics. This level of efficiency in transfection allows for cellular studies, including genome editing using CRISPR and ZFNs.

\section{Conclusion}

The method described here is the first reported for the transient expression of heterologous genes in Cannabis protoplasts. The variables involved in protoplast isolation were verified on three cultivars with varying cannabinoid content for protocol robustness. The transfection protocol was optimised for a high THC yielding strain within mesophyll protoplasts. This method can be easily adapted for transient expression studies using CRISPR/Cas-9, protein-protein interaction or other investigations in Cannabis where a transient gene expression system is desired.

Supplementary Information The online version contains supplementary material available at https://doi.org/10.1007/s11627-021-10178-0.

Acknowledgements Authors acknowledge Canopy Growth Corporation for access to plant material to evaluate the protocols, where applicable. 
The authors also wish to acknowledge Navdeep Kaur for FACS operation and optimisation.

Author contribution LMO and EM in protocol development and optimisation. LMO assembled the manuscript.

Funding Funding was provided by Agriculture Victoria Services and Agriculture Victoria Research.

\section{Declarations}

Conflict of interest The authors declare no competing interests.

Open Access This article is licensed under a Creative Commons Attribution 4.0 International License, which permits use, sharing, adaptation, distribution and reproduction in any medium or format, as long as you give appropriate credit to the original author(s) and the source, provide a link to the Creative Commons licence, and indicate if changes were made. The images or other third party material in this article are included in the article's Creative Commons licence, unless indicated otherwise in a credit line to the material. If material is not included in the article's Creative Commons licence and your intended use is not permitted by statutory regulation or exceeds the permitted use, you will need to obtain permission directly from the copyright holder. To view a copy of this licence, visit http://creativecommons.org/licenses/by/4.0/.

\section{References}

Braich S, Ballie R, Jewell L, Spangenberg G, Cogan N (2019) Generation of a comprehensive transcriptome atlas and transcriptome dynamics in medicinal cannabis. Sci Rep 9:1-12

Chawla HS (2011) Introduction to plant biotechnology, vol 3. Science Publishers, Enfield New Hampshire

Chen J, Yi Q, Song Q, Gu Y, Zhang J, Hu Y, Liu H, Liu Y, Yu G, Hang Y (2015) A highly efficient maize nucellus protoplast system for transient gene expression and studying programmed cell death-related processes. Plant Cell Rep 34:1239-1251

Clough SJ, Bent AF (1998) Floral dip: a simplified method for Agrobacterium-mediated transformation of Arabidopsis thaliana. Plant J 16:735-743

Deguchi M, Bogush D, Weeden H, Spuhler Z, Potlakayala S, Kondo T, Zhang Z, Rudrabhatla S (2020) Establishment and optimization of a hemp (Cannabis sativa L.) agroinfiltration system for gene expression and silencing studies. Sci Rep 10:1-11

Douchkov D, Nowara D, Zierold U, Schweizer P (2005) A highthroughput gene-silencing system for the functional assessment of defense-related genes in barley epidermal cells. Mol Plant Microbe Interact 18:755-761

Feeney M, Punja ZK (2003) Tissue culture and Agrobacterium-mediated transformation of hemp (Cannabis sativa L.). In Vitro Cell Dev Biol - Plant 39:578-585

Feeney M, Punja ZK (2017) The role of Agrobacterium-mediated and other gene-transfer technologies in Cannabis research and product development, Chandra, S., Lata, H., ElSohly, M. (eds), Cannabis sativa L.-Botany and Biotechnology, vol 1, Springer, Cham. pp. 343-363

Fischer R, Hain R (1995) Tobacco protoplast transformation and use for functional analysis of newly isolated genes and gene constructs. In: Galbraith DW, Bourque DP, Bohnert HJ (eds) Methods in Cell Biology, vol 50. Elsvier, pp 401-410
Furze JM, Rhodes MJC, Robins RJ (1987) The use of agarose bead culture for the regeneration of single cell-derived colonies from protoplasts isolated from suspension cultures of Humulus lupulus. Plant Cell Tiss Org Cult 8:17-25

Gagne SJ, Stout J, Liu E, Boubakir Z, Clark S, Page J (2012) Identification of olivetolic acid cyclase from Cannabis sativa reveals a unique catalytic route to plant polyketides. Proc Natl Acad Sci 109:12811-12816

Grassa CJ, Wenger JP, Dabney C, Poplawski SG, Motley ST, Michael TP, Schwartz CJ, Weiblen GD (2018) A complete Cannabis chromosome assembly and adaptive admixture for elevated cannabidiol (CBD) content. bioRxiv:458083. https://doi.org/10.1101/458083

Gurunani SG, Gholse YN, Yeole MP, Chaple DR (2015) Hairy root culture: an experimental system for secondary metabolite production. Res J Pharm Tech 8:728-730

Heale JB, Legg T, Connell S (1989) Humulus lupulus L.(Hop): In vitro culture; Attempted production of bittering components and novel disease resistance. In: Bajaj YPS (ed) Medicinal and Aromatic Plants II, Biotechnology in Agriculture and Forestry, vol 7. Springer, Berlin, Heidelberg, pp 264-285

Jeon JM, Ahn NY, Son BH, Kim CY, Han C, Kim G, Gal SW, Lee S (2007) Efficient transient expression and transformation of PEGmediated gene uptake into mesophyll protoplasts of pepper (Capsicum annuum L.). Plant Cell Tiss Org Cult 88:225-232

Jia X, Zhang X, Qu J, Han R (2016) Optimization conditions of wheat mesophyll protoplast isolation. Agri Sci 7:850-858

Jones RL (1979) Cell culture, protoplast isolation, and cell fusion of Cannabis Sativa L.: evaluation of chilling preventative chemicals and quality control of bananas in the tropics, University of Houston Central Campus, M. Sc. Thesis, DOI: 10.13140/ RG.2.2.19104.43527

Kuriakose B, Du Toit ES, Jordaan A (2012) Transient gene expression assays in rose tissues using a Bio-Rad Helios hand-held gene gun. $\mathrm{S}$ Afr J Bot 78:307-311

Laverty KU, Stout J, Sullivan MJ, Shah H, Gill N, Holbrook L, Deikus G, Sebra R, Hughes TR, Page JE, van Bakel H (2019) A physical and genetic map of Cannabis sativa identifies extensive rearrangements at the THC/CBD acid synthase loci. Genome Res 29:146-156

Leffel SM, Mabon SA, Stewart CN Jr (1997) Applications of green fluorescent protein in plants. Biotechniques 23:912-918

Lu J, Bai M, Ren H, Liu J, Wang C (2017) An efficient transient expression system for gene function analysis in rose. Plant Method 13:116

Maas C, Werr W (1989) Mechanism and optimized conditions for PEG mediated DNA transfection into plant protoplasts. Plant Cell Rep 8: $148-151$

Marion J, Bach L, Bellec Y, Meyer C, Gissot L, Faure J (2008) Systematic analysis of protein subcellular localization and interaction using high-throughput transient transformation of Arabidopsis seedlings. Plant J 56:169-179

Matchett-Oates L, Braich S, Spangenberg G, Rochfort S, Cogan N (2020) In silico enablinginformed design for genome editing in medicinal cannabis, gene families and variant characterisation. Plant Methods. IN REVIEW

Morimoto S, Tanaka Y, Sasaki K, Tanaka H, Fukamizu T, Shpyama Y, Shoyama Y, Taura F (2007) Identification and characterization of cannabinoids that induce cell death through mitochondrial permeability transition in Cannabis leaf cells. J Biol Chem 282:20739 20751

Murashige T, Skoog F (1962) A revised medium for rapid growth and bio assays with tobacco tissue cultures. Physiol Plant 15:473-497

Nanjareddy K, Arthikala M, Blanco L, Arellano E, Lara M (2016) Protoplast isolation, transient transformation of leaf mesophyll protoplasts and improved Agrobacterium-mediated leaf disc infiltration of Phaseolus vulgaris: tools for rapid gene expression analysis. BMC Biotechnol 16:53 
Nicolaisen M, Poulsen GB (1993) Optimization of polyethylene glycol mediated transient gene expression in pea protoplasts. Plant Cell Tiss Org Cult 35:93-97

Page MT, Parry MAJ, Carmo-Silva E (2019) A high-throughput transient expression system for rice. Plant Cell Environ 42:2057-2064

Priyadarshani S, Hu B, Li W, Ali H, Jia H, Zhao L, Ojolo SP, Azam SM, Xiong J, Yan M, Rahman ZU, Wu Q, Qin Y (2018) Simple protoplast isolation system for gene expression and protein interaction studies in pineapple (Ananas comosus L.). Plant Method 14:95

ProCon.org (2021) Legal Medical Marijuana States and DC. Available at: https://medicalmarijuana.procon.org/legal-medical-marijuanastates-and-dc/ (Accessed: 24 March 2021).

Raharjo TJ, Cheng W, Verberne MC, Peltenburg-Looman AMG, Linthorst HJM, Verpoorte R (2004) Cloning and over-expression of a cDNA encoding a polyketide synthase from Cannabis sativa. Plant Physiol and Biochem 42:291-297

Rasmussen JO, Rasmussen OS (1993) PEG mediated DNA uptake and transient GUS expression in carrot, rapeseed and soybean protoplasts. Plant Sci 89:199-207

Reed J, Osbourn A (2018) Engineering terpenoid production through transient expression in Nicotiana benthamiana. Plant Cell Rep 37: $1431-1441$

Saenger W, Hunter WN, Kennard O (1986) DNA conformation is determined by economics in the hydration of phosphate groups. Nature 324:385-388

Sain PM, Rodríguez S, Disalvo EA (2017) Effect of osmotic stress on the surface properties of protoplasts as measured by merocyanine 540 . Soc Plant Res 30:75-80

Sheen J (2001) Signal transduction in maize and Arabidopsis mesophyll protoplasts. Plant Physiol 127:1466-1475

Shen Y, Meng D, McGrouther K, Zhang J, Cheng L (2017) Efficient isolation of Magnolia protoplasts and the application to subcellular localization of MdeHSF1. Plant Method 13:44

Shoyama Y, Yagi M, Nishioka I, Yamauchi T (1975) Biosynthesis of cannabinoid acids. Phytochem 14:2189-2192

Sirikantaramas S, Morimoto S, Shoyama Y, Ishikawa Y, Wada Y, Shoyama Y, Taura F (2004) The gene controlling marijuana psychoactivity molecular cloning and heterologous expression of Delta1-tetrahydrocannabinolic-acid synthase from Cannabis sativa. J Biol Chem 279:39767-39774

Smirnoff N (2019) Engineering of metabolic pathways using synthetic enzyme complexes. Plant Physiol 179:918-928
Su C, Wang Y, Hsieh T, Lu C, Tseng T, Yu S (2010) A novel MYBS3dependent pathway confers cold tolerance in rice. Plant Physiol 153: $145-158$

Taura F, Morimoto S, Shoyama Y (1996) Purification and characterization of cannabidiolic-acid synthase from Cannabis sativa L. Biochemical analysis of a novel enzyme that catalyzes the oxidocyclization of cannabigerolic acid to cannabidiolic acid. J Bio Chem 271:17411-17416

Taura F, Sirikantaramas S, Shoyama Y, Yoshikai K, Shoyama Y, Morimoto S (2007) Cannabidiolic-acid synthase, the chemotypedetermining enzyme in the fiber-type Cannabis sativa. FEBS Lett 581:2929-2934

Taura F, Tanaka S, Taguchi C, Fukamizu T, Tanaka H, Shoyama Y, Morimoto S (2009) Characterization of olivetol synthase, a polyketide synthase putatively involved in cannabinoid biosynthetic pathway. FEBS Lett 583:2061-2066

Töpfer R, Pröls M, Schell J, Steinbiß HH (1988) Transient gene expression in tobacco protoplasts: II. Comparison of the reporter gene systems for CAT, NPT II, and GUS. Plant Cell Rep 7:225-228

Vain P, McMullen MD, Finer JJ (1993) Osmotic treatment enhances particle bombardment-mediated transient and stable transformation of maize. Plant Cell Rep 12:84-88

Van Bakel H, Stout J, Cote A, Tallon C, Sharpe A, Hughes T, Page J (2011) The draft genome and transcriptome of Cannabis sativa. Genome Biol 12:R102

Wahby I, Caba JM, Ligero F (2013) Agrobacterium infection of hemp (Cannabis sativa L.): establishment of hairy root cultures. J Plant Interact 8:312-320

Wang YC, Klein TM, Fromm M, Cao J, Sanford JC, Wu R (1988) Transient expression of foreign genes in rice, wheat and soybean cells following particle bombardment. Plant Mol Biol 11:433-439

Yoo SD, Cho YH, Sheen J (2007) Arabidopsis mesophyll protoplasts: a versatile cell system for transient gene expression analysis. Nat Prot 2:1565-1572

Zhang Y, Su J, Duan S, Ao Y, Dai J, Liu J, Wang P, Li Y, Liu B, Feng D, Wang J, Wang H (2011) A highly efficient rice green tissue protoplast system for transient gene expression and studying light/ chloroplast-related processes. Plant Method 7:30-38

Zuardi AW (2006) History of cannabis as a medicine: a review. Braz J Psychiatry 28:153-157 